\title{
Río de Janeiro como smart city: ciudadanía y participación en la "polisdigitocracia"
}

\author{
Paulo César Castro \\ Universidade Federal do Rio de Janeiro, Brasil \\ paulo.castro@eco.ufri.br \\ Recibido: 31/7/2018 / Aceptado: 21/10/2018 \\ doi: 10.26439/contratexto2019.n031.3897
}

Resumen. En el 2013 Río de Janeiro fue elegida la Ciudad Inteligente del año por la Smart City Expo World Congress, una feria celebrada en Barcelona. La capital del estado de Río de Janeiro se destacó con el proyecto Gestión de Alto Desempeño, que incluye el subproyecto Centro de Operaciones Río (COR). El COR es un centro de monitoreo que tiene 1500 cámaras y analiza datos recibidos en tiempo real de varios sensores tecnológicos repartidos por la ciudad, resultado de un acuerdo entre el ayuntamiento y varias empresas de tecnología. La iniciativa es defendida como parte de la creencia en un modelo de "polisdigitocracia", palabra que, según el exalcalde Eduardo Paes, significa la versión digital de la democracia griega para las ciudades del siglo xxI. Este artículo parte de una investigación en curso; pretende evaluar - a través de los discursos de los actores directamente involucrados con el proyecto- cómo se puso en práctica la idea de smart city en Río. La investigación tiene como objetivo analizar si la tecnología está siendo usada como una estrategia para que la ciudad tenga sus destinos definidos por los expertos o si en la palabra "inteligencia" cabe también el conocimiento de los habitantes.

Palabras clave: smart city / Río de Janeiro / polisdigitocracia / tecnología / comunicación 


\section{Rio de Janeiro as a smart city: citizenship and participation in "polisdigitocracy"}

Авstract. Rio de Janeiro was elected in 2013 as the "Smart City of the Year" by the Smart City Expo World Congress, a fair held in Barcelona. The capital of the state of Rio de Janeiro was highlighted with the project Gestão de Alto Desempenho (High Performance Management) which includes the subproject Centro de Operações Rio (COR - Rio Operations Center). COR is a monitoring center that has 1,500 video cameras and analyzes data received in real time from several technological sensors scattered throughout the city. This is the result of an agreement between the city hall and several technology companies. The initiative is supported as part of the belief in a model of "polisdigitocracy", a word that, according to former Mayor Eduardo Paes, means the digital version of Greek democracy for cities in the 21st century. This article is based on an ongoing investigation. It intends to evaluate - through discourses of the actors directly involved in the project- how the idea of smart city in Rio was put into practice. The research aims to analyze if technology is being used as another strategy for the city to have its destiny defined by the experts or if the word "intelligence" also includes the knowledge of the residents. Keywords: smart city / Rio de Janeiro / polisdigitocracy / technology / communication 


\section{Introducción}

7 uando Río de Janeiro fue elegida la ciudad más inteligente del mundo en 2013, por la Smart City Expo World Congress, una feria sobre smart cities celebrada en Barcelona, se destacó la expresión "polisdigitocracia". El neologismo fue creado por el exalcalde de la ciudad, Eduardo Paes, para representar su visión sobre el modo en que la "ciudad maravillosa" debería enfrentar sus problemas, históricamente persistentes, con ayuda de las tecnologías digitales. La polis digital de la cabeza del ex gobernante se remite a Grecia de 2800 años atrás, donde la participación de los ciudadanos en el destino de las ciudadesestado era decisiva. Él piensa que, así como los griegos antiguos, los cariocas pueden ser también los actores principales en las soluciones de los problemas de ciudades tan populosas como Río de Janeiro, no limitándose solo a la participación en el proceso electoral. Paes defiende que tal condición de actuación está dada por la omnipresencia de los dispositivos digitales, ya debidamente incorporados por los ciudadanos en sus conexiones y comunicaciones.

Pero, ¿los residentes están efectivamente siendo partícipes de la construcción de Río de Janeiro como smart city? Con base en la recolección y procesamiento de datos de diferentes orígenes y formatos, incluso los generados por los propios ciudadanos, ¿a partir de qué lugar la ciudad inteligente en la que pretende convertirse Río considera a los residentes? ¿Solo como "donantes" pasivos de datos, a menudo sin siquiera tener conciencia o noción del volumen de esa apropiación de sus informaciones por la administración pública, como ya ocurre en las redes sociales? ¿Hasta qué punto el Ayuntamiento está repitiendo un modelo cuyos escándalos actuales, principalmente los relacionados con Facebook, han demostrado la urgencia del debate sobre la transparencia en la forma en que se recopilan los datos de los usuarios?

Estas cuestiones orientan el texto que sigue, cuyo objetivo principal es evaluar los discursos de los actores involucrados directamente con el proyecto de ciudad inteligente para Río de Janeiro. Pero antes de llegar específicamente a ellas, inicialmente se hace una rápida revisión sobre la formación de las primeras ciudades, buscando evaluar la importancia fundamental de la tecnología, principalmente de la escritura, para lo que fue llamado Revolución Urbana. Considerados los debidos contextos que separan las ciudades sumerias de las de hoy, distanciadas por casi 7500 años, en el movimiento actual de estructuración de las smart cities la tecnología, fundamentalmente digital, ha ejercido, de la misma forma, un papel crucial.

Enseguida, Río de Janeiro es presentada en algunas de sus peculiaridades, ostentando la posición de segunda mayor ciudad de Brasil, con sus más de 6,5 millones de habitantes. La capital del Estado de Río de Janeiro puede ser 
caracterizada por sus bellezas naturales, combinación de playas y montañas, pero también por sus contrastes sociales y económicos. La apuesta excesiva en la tecnología como salida de esos obstáculos, con inversión más en el smart que en el sensible, puede no resultar verdaderamente en la "polisdigitocracia" que el exalcalde defiende.

\section{Ciudades y tecnologías de la información y comunicación: una relación de mutua dependencia hace unos 7500 años}

Si las ciudades están pasando por profundas transformaciones, estas pueden explicarse, entre otros factores, por la prominencia que las tecnologías digitales han asumido en los últimos 40 años en todas las instancias de la vida social. Pero una vuelta en el tiempo va a mostrar que el surgimiento de las primeras ciudades en el mundo, aproximadamente en 5400 a. C., tuvo como una de sus condiciones fundamentales también a las tecnologías. Fue más precisamente en Sumeria, al sur de Mesopotamia (en la región del actual Irak), que surgió el primer asentamiento urbano que puede ser llamado ciudad, Eridu. Y con esa colectividad comunitaria, formada alrededor principalmente de la agricultura y del comercio, estaba dado el paso inicial de lo que fue llamado por Gordon Childe (1950, pp. 9-16) Revolución Urbana. El arqueólogo australiano incluso estableció 10 criterios para distinguir las primeras ciudades de cualquier pueblo, antiguo o incluso contemporáneo. Vale la pena enumerarlos brevemente:

1. Grandes y densas poblaciones (aunque, comparadas con las de nuestros días, las ciudades sumerias, con poblaciones de entre 7000 y 20000 habitantes, podrían ser clasificadas apenas como un barrio de cualquier ciudad de tamaño medio actual).

2. Especialización de la mano de obra, organizada a través de los campesinos ocupados en la agricultura, la actividad pecuaria, la pesca o la recolección; de los artesanos especializados a tiempo completo; de los trabajadores de transporte, comerciantes, oficiales y sacerdotes.

3. Sociedad estructurada en clases incluyendo una clase dominante formada por todos los que no estaban involucrados en la producción de alimentos, como sacerdotes, líderes civiles y militares y funcionarios al servicio de la administración.

4. Sistema de tasación a través del cual cada agricultor pagaba sobre su producción de alimentos un excedente, como diezmo o impuesto, para una divinidad imaginaria o un rey divino. 
5. Arquitectura monumental que distingue a las ciudades de las aldeas los edificios públicos imponentes, principalmente templos y lugares donde se concentraban los excedentes.

6. La presencia del gobierno, definido por los métodos de administración de la ciudad, principalmente de los excedentes, de la construcción de edificios, la remuneración de funcionarios, pero también a través de la institución de leyes (reglas, normas) a ser seguidas por la comunidad;

7. Invención de la escritura y de la notación numérica como métodos convencionales al servicio del registro de los excedentes, de la población, del sacerdocio y de los relatos míticos e históricos, debiendo ser inteligibles para todos los que se ocupaban de esas tareas y sus sucesores, y sustitutos seguros de la memoria típica de las comunidades orales.

8. El conocimiento científico, establecido a partir de ciencias exactas y predictivas como la aritmética, la geometría y la astronomía, que tuvieron sus condiciones de surgimiento a partir de la escritura y de la disponibilidad de los funcionarios ociosos, aquellos libres de la producción de alimentos.

9. Comercio regular a largas distancias de materias primas no disponibles localmente necesarias a la industria para los cultos y pagadas con parte de los excedentes.

10. Arte monumental practicado por escultores, pintores o grabadores a tiempo completo, a través de una expresión artística orientada por estilos sofisticados, diferenciados del naturalismo ingenuo de los cazadores-recolectores.

Todos los elementos aquí relacionados todavía sirven muy bien a la definición contemporánea de ciudad, siempre que a ella se agreguen, por supuesto, otros aspectos y sean considerados los niveles adecuados de complejidad -sociales, culturales, económicos, políticos, comunicacionales, urbanísticos, tecnológicos, etcétera- a que ha sido históricamente sometida. En el ámbito de este artículo no cabe la discusión de todos ellos, pero merece destacar la importancia vital que la escritura tuvo como tecnología de la comunicación y de la información ${ }^{1}$ no solo para la estructuración de la ciudad sino principalmente como marca de la civilización, como defienden Childe (1950) y otros autores (Townsend, 2013).

1 Pierre Lévy (1993) considera que las tecnologías de la información y la comunicación, como la escritura, también son tecnologías de la inteligencia, y que los dispositivos técnicos son aparatos de memoria. 
La invención de este dispositivo como sistema de registro de los más variados aspectos de la urbanidad, como recurso auxiliar para la memoria, y de otros capaces de propiciar exactitud y predicción, guarda proximidad, hechas las debidas salvedades, con el movimiento que hoy va conformando a las smart cities.

Las ciudades, por lo tanto, en toda la historia de su funcionamiento, siempre fueron dependientes de técnicas y métodos que permiten catalogar, inventariar, relacionar, registrar, almacenar, tratar, recuperar y transmitir los datos generados por ellas mismas, como instancias administrativas y políticas, pero también por sus ciudadanos a través de las diferentes actividades ejercidas por ellos. Es evidente que las ciudades también pueden ser tomadas como los ambientes más propicios para la invención de tecnologías como la escritura y de otros tipos, instaurando así una relación de mutua dependencia entre las dos entidades.

Transcurridos casi 7500 años desde el inicio de la revolución urbana, crecieron exponencialmente las ciudades, los datos y las tecnologías para registrarlos, administrarlos y procesarlos. Y con ellos también aumentaron los problemas, con los cuales los sumerios y egipcios probablemente ni siquiera imaginaban que un día fuese necesario lidiar. Las ciudades ya no están hechas de hormigón, acero y vidrio, sino también de una gran cantidad de dispositivos gestionados por software y algoritmos y conectados por internet. Se creyó que la conectividad instantánea y omnipresente generada por internet llevaría a la pérdida de importancia del espacio físico para las relaciones sociales y, con ello, a la muerte de las ciudades (Karlgaard y Malone, 1995).

Las ciudades continúan creciendo: según datos de la Organización de las Naciones Unidas (2014), más de la mitad de la población mundial (54 \%) vive en áreas urbanas. El número representa un crecimiento del $30 \%$ con respecto a 1950. Y en 2050 el número puede crecer al $66 \%$. Ante las grandes dificultades por las que pasan las ciudades en las más diferentes áreas, hay quienes defienden que están disponibles, en contrapartida, grandes soluciones, confiadas principalmente a las tecnologías digitales. Para casi todos los problemas de las ciudades contemporáneas en diferentes sectores, una o más salidas con aplicación de los recursos tecnológicos son señaladas.

La definición de smart city está íntimamente asociada a nociones como big data (grandes volúmenes de datos), redes en nube, dispositivos móviles, Internet de los Objetos (equipos automatizados sensibles al medio ambiente, en comunicación digital entre sí), algoritmos y todo lo que está relacionado con el universo de internet. Tal momento es el ápice de un proceso histórico que progresivamente asoció la expansión y el progreso de las ciudades con las tecnologías de la información y la comunicación (TIC). El siglo XIX, por ejemplo, fue también testigo de ese desarrollo urbano a través del telégrafo y del ferrocarril. Las dos invenciones 
son responsables del aumento de la capacidad de procesamiento de la información y comunicación de forma rápida y a largas distancias. Pero si antes el uso y la posesión de las TIC era limitado por una serie de factores, los ciudadanos de hoy, portando sus diferentes dispositivos (smartphones, tablets, e-readers, gafas, relojes y ropas conectadas), se transforman en verdaderos sensores móviles por la ciudad (y por el mundo), no solo recibiendo sino también produciendo, voluntaria o involuntariamente, gigantescos volúmenes de datos. "La ciudad pasa a ser un organismo informacional que reacciona y actualiza a todos sobre sus condiciones en cualquier momento" ${ }^{\prime 2}$. (Lemos, 2014)

¿Qué significa exactamente la smart city? ¿Las soluciones tecnológicas son las mismas para todas las ciudades, independientemente de su tamaño (megalópolis, metrópolis, medianas, pequeñas), su ubicación en el mundo; de si nacieron planificadas o no, de sus diferentes funciones (político-administrativas, industriales, portuarias, religiosas, históricas, turísticas, tecnológicas); si pasaron por el proceso de conurbación (cuando una ciudad se expandió tanto horizontalmente en sus límites geográficos que se mezcló con otras áreas urbanas, estableciendo una región metropolitana); de sus diferentes propuestas de crecimiento y desarrollo; de si son ricas o pobres, entre otros factores? Consideremos la definición que se encuentra en el estudio titulado Smart City: essentials for city leaders, producido por el Global Center for Digital Business Transformation, iniciativa del International Institute for Management Development (IMD) y Cisco:

Una Ciudad Inteligente es un área urbana que se ha vuelto más eficiente y/o más amigable con el medio ambiente y/o más inclusiva socialmente a través del uso de tecnologías digitales. El objetivo de una Smart City es mejorar su atractivo para los ciudadanos y/o las empresas mejorando o agregando servicios de la ciudad 3 . (Pfaelli, Rollier, Vonlanthen, \& Wade, 2016, p. 6)

El documento indica además que los "servicios de la ciudad" (city services) pueden ser tomados como algo que la ciudad ofrece a sus ciudadanos o a los negocios, incluyendo iluminación, gestión del tráfico, estacionamiento público, electricidad, etcétera.

En el proyecto que los autores llaman "Smart City Piano", producido a partir de entrevistas con representantes de 25 ciudades del mundo (incluyendo stakeholders,

2 "A cidade passa a ser um organismo informacional que reage e atualiza todos sobre suas condições a qualquer hora".

3 "A Smart City is an urban area that has become more efficient and/or more environmentally friendly and/or more socially inclusive through the use of digital technologies. The goal of a Smart City is to improve its attractiveness to citizens and/or businesses by enhancing or adding city services". 
proveedores de productos y servicios y responsables de las compras de organizaciones y departamentos de las ciudades), las motivaciones de una ciudad para convertirse en smart se deben mover por los siguientes criterios: 1 . beneficios de la eficiencia; 2. objetivos ambientales; 3 . aumento de la inclusión social, y 4. aumento del atractivo de la ciudad. Los ejemplos de smart cities citados utilizan las tecnologías digitales basadas en la recolección y la disponibilidad de datos para aumentar los ingresos, reducir costos, agilizar procesos, mantener empresas y atraer nuevas, reducir la contaminación y el tráfico. Sin embargo, el estudio es muy tímido al relatar las experiencias para expandir la inclusión de los ciudadanos. Este, por cierto, es el aspecto que menos gana atención y resulta difícil entender lo que el proyecto de una smart city puede buscar mejorar para incluir en la ciudad a sus habitantes.

La poca claridad sobre la forma en que los datos de los ciudadanos están siendo utilizados por la administración de la ciudad o por empresas y organizaciones que tienen acceso a los datos públicos, puede ser un gran problema para la adhesión y el compromiso de los residentes en el proyecto. Pero hay quien cree que, en el proceso de implementación de los proyectos de smart cities a partir de la ubicuidad de la tecnología digital, el ciudadano puede ser más que un mero coadyuvante.

"Ya no eres solo un engranaje de una gran máquina. Eres parte de la mente de la ciudad inteligente en sí misma. Y eso te da poder para dar forma al futuro"4 (Townsend, 2013, p. xiii). Townsend es más optimista aún al decir que los smartphones se han convertido en una plataforma para reinventar las ciudades de arriba hacia abajo y que son la parte visible del nacimiento de un nuevo movimiento cívico (2013, p. xiv).

Una visión diferente de la noción de smart city es adoptada por el Senseable City Laboratory, iniciativa de investigación del Massachussets Institute of Technology (MIT). A través de ella, la idea es que el foco sea más en los ciudadanos que en la tecnología y que esta sea solo un facilitador del propósito de aumentar la calidad de vida en las ciudades.

\section{Río de Janeiro de los límites: de la bonanza a los problemas de una gran ciudad}

Como la mayoría de las grandes ciudades brasileñas, Río de Janeiro ostenta, dependiendo del aspecto por el cual es evaluada, cifras dignas de elevarla a las más altas consideraciones y, en el otro polo, estadísticas capaces de colocarla al lado de los rincones más atrasados del planeta. Aunque el Índice de Desarrollo Humano

4 "You are no longer just a cog in a vast machine. You are part of the mind of the smart city itself. And that gives you power to shape the future". 
(IDH) de la ciudad, de 0,799 (Instituto Brasileiro de Geografia e Estatística, 2010), sea medio, muy cerca de lo alto, los contrastes entre riqueza y pobreza conviven lado a lado, exhibiendo la cara cruel de la desigualdad social y económica. Cuando se trata de la telefonía celular, por ejemplo, Río exhibe resultados dignos de ciudades de alto poder adquisitivo. La ciudad forma parte del Estado con la tercera mayor densidad de líneas móviles (pre y pospagadas) por cada 100 habitantes de Brasil $(124,75)$, quedando atrás solo del Distrito Federal $(162,57)$, donde se encuentra Brasilia, y del Estado de São Paulo $(138,12)$. Como Río tiene una población equivalente a casi el $40 \%$ del Estado (6 520 266), es posible estimar que la densidad de celulares por habitante sea aún mayor. Río sigue la tendencia vista en prácticamente todas las grandes ciudades del país, en las que el número de celulares es mayor que la población.

Pero si el indicador observado es el tránsito, la ciudad ocupa la primera posición entre los municipios brasileños (y la octava del mundo) en que sus habitantes más consumen tiempo en embotellamientos, según datos del 2017 (Río de Janeiro é uma das 10 cidades mais congestionadas do mundo, diz pesquisa, 2017; Bruwer, 2010). Pero no es para menos: con una flota de 2,73 millones de vehículos (coches, motocicletas, autobuses, camiones y otros), hay en Río un coche para cerca de 2,4 habitantes, situación que es aún más agravada debido a la topografía de la ciudad, altamente accidentada, y por la baja calidad del sistema público de transporte, a pesar de las mejoras realizadas en los últimos tres años, como la implantación de sistemas de Bus Rapid Transport (BRT) y Vehículo Ligero sobre Rieles (Veículo Leve sobre Trilhos - VLT). Se puede incluir también como factor a contribuir en los constantes embotellamientos, principalmente en las horas de ida al trabajo y vuelta a casa, la concentración de las mejores opciones de empleo y ocio en el centro y en la zona sur de la ciudad (la última es la más noble de todas). Es un desplazamiento que involucra a las poblaciones de otras 20 ciudades de la Región Metropolitana, totalizando, con Río, 12,3 millones de habitantes. La alerta del arquitecto y urbanista Carlos Leite sobre la cuestión es enfática.

Aproximadamente dos millones de personas todos los días salen de sus vi-
viendas en la Región Metropolitana de Río de Janeiro para ir a trabajar en el
centro ampliado de la capital. Perdimos valiosas dos horas de vida para hacer
ese movimiento pendular. En el lugar que los acoge para trabajar, abundan
oportunidades de trabajo y económicas. Sólo el 2,6 \% de la población vive allí.
Es decir, tenemos una total desconexión entre el lugar de vivir y trabajar. De
nada servirá construir miles de kilómetros de BRT y VLT si esa dinámica no se
resuelve ${ }^{5}$. (Leite, 2014, p. 54)

5 "Aproximadamente dois milhões de pessoas todos os dias saem de suas moradias na Região Metropolitana do Rio de Janeiro para ir trabalhar no centro ampliado da capital. 
Otro paisaje en que la fotografía de Río no queda muy hermosa es el de la violencia urbana. Aunque en los últimos años la ciudad haya desaparecido de la lista de municipios más violentos de Brasil - de acuerdo con el Atlas de la Violencia 2017 del Instituto de Pesquisa Econômica Aplicada (IPEA) (Cerqueira et al., 2017, pp. 61-68) - e incluso de la lista de las 50 ciudades más violentas del mundo - segundo ranking de la organización mexicana Seguridad, Justicia y Paz (Las 50 ciudades más violentas, 2017) - , la explicación está en el hecho de que la violencia se extendió hacia otras regiones del país, principalmente las capitales del Nordeste y ciudades menores del Norte. Pero después de la caída acentuada de la tasa de homicidios en la ciudad entre el 2010 y el 2015 (de 43,0 a 22,3 por 100 mil habitantes, con el pico de 70,6 en 1999), resultado de la implantación de las Unidades de Policía Pacificadora (UPP), las principales favelas y el aumento del contingente de policías, las cifras volvieron a crecer en el $2016(29,4)$ y el 2017 $(32,0)$. Es importante recordar dos aspectos en esta cuestión: en Brasil la lucha contra la violencia urbana es atribución de los estados, a través de sus policías militar y civil, sin embargo, los ayuntamientos pueden desempeñar un papel importante en esa área por la forma en que definen la política de acción de sus guardias municipales; las tasas por encima de 10 homicidios por 100 mil habitantes son consideradas por la ONU atestados de violencia epidémica.

La tabla 1 presenta un poco más de Río de Janeiro con cifras que dan la dimensión de los límites en que se encuentran la ciudad y sus habitantes.

Tabla 1

Río de Janeiro en números

\begin{tabular}{|l|c|}
\hline \multicolumn{2}{|c|}{ Río de Janeiro en números } \\
\hline Población estimada (2017) & 6520266 \\
\hline Población en el último censo (2010) & 6320446 \\
\hline Densidad demográfica hab/km² (2010) & 5265,82 \\
\hline Indice de Desarrollo Humano - IDH (2010) & 0,799 \\
\hline \multicolumn{2}{|c|}{ Educación } \\
\hline $\begin{array}{l}\text { Tasa de escolarización de 6 a 14 años de edad } \\
\text { [2010) }\end{array}$ \\
\hline
\end{tabular}

(continúa)

Perdem valiosas duas horas de vida para fazer esse movimento pendular. No lugar que os acolhe para trabalhar, abundam oportunidades de trabalho e econômicas. Apenas 2,6\% da população mora ali. Ou seja, temos uma total desconexão entre lugar de morar e trabalhar. De nada adiantará construir milhares de quilômetros de BRTs e VLTs se essa dinâmica não for reequacionada". 


(continuación)
\begin{tabular}{|l|c|}
\hline \multicolumn{2}{|c|}{ Salud } \\
\hline $\begin{array}{l}\text { Mortalidad infantil (muertes por mil nacidos vivos) } \\
(2014)\end{array}$ & 11,32 \\
\hline \multicolumn{2}{|c|}{ Territorio y medio ambiente } \\
\hline Área de la unidad territorial en $\mathrm{km}^{2}$ (2016) & 1200,177 \\
\hline Tratamiento sanitario adecuado (2010) & $94,4 \%$ \\
\hline Arborización de vías públicas & $70,50 \%$ \\
\hline Urbanización de vías públicas & $78,40 \%$ \\
\hline
\end{tabular}

Fuente: Instituto Brasileiro de Geografia e Estatística, 2018

\section{"Polisdigitocracia": la versión digital de la democracia griega para las ciudades del siglo XXI}

Fue esta Río de Janeiro de acentuados contrastes económicos y sociales, a través de los aspectos aquí mostrados y de otros, que en el 2013 fue elegida la Ciudad Inteligente del año por la Smart City Expo World Congress. Una investigación informal con algunos residentes de la capital del Estado de Río de Janeiro, la mayoría con amplio acceso a informaciones, mostró que la reacción a la pregunta sobre la conquista del premio era siempre de incredulidad, a veces seguida de un complemento de ironía. Para quienes viven cotidianamente en la ciudad, es casi imposible creer que Río de Janeiro sea una smart city frente a tantos problemas relacionados con el tránsito, la violencia y el desorden urbano, las atenciones de salud, las consecuencias que provienen de lluvias y vendavales fuertes, entre otros.

Aunque los cariocas son escépticos en cuanto a la inteligencia aplicada a la administración de la ciudad, Río se destacó mundialmente con el proyecto Gestión de Alto Desempeño, en el cual está asignado el subproyecto Centro de Operaciones Río (COR). Integrando las actividades de 30 órganos (secretarías municipales y concesionarias de servicios públicos), el COR es un centro de monitoreo que dispone de 1500 cámaras (800 propias y 700 de concesionarias y de la Secretaría de Seguridad Pública del Estado) y analiza datos recibidos en tiempo real de varios sensores tecnológicos repartidos por la ciudad. Funciona 24 horas al día, siete días a la semana, con un cuerpo de más de 500 profesionales trabajando en diferentes turnos.

Desde que fue creado en el 2010 tiene como objetivo "anticipar soluciones, alertando a los sectores responsables sobre los riesgos y las medidas urgentes que deben ser tomadas en casos de emergencia" (COR completa seis anos, Prefeitura de Río de Janeiro, 2016). El Centro es el resultado de un acuerdo entre 
el Ayuntamiento e IBM, pero involucra, entre otras, empresas de tecnología como Cisco, Mauell, Oi (telefonía) y Samsung. Es importante señalar que el COR fue creado durante la gestión del alcalde Eduardo Paes (2009-2016) y que actualmente, siendo la ciudad administrada por un nuevo alcalde, Marcelo Crivella, la dirección del proyecto ha sido cambiada, solo en 2017, cuatro veces.

Como parte del proceso que busca transformar Río en una smart city, el Ayuntamiento ha editado un decreto en 2013 para crear el área de Big Data de la ciudad, llamada "Pensa - Sala de Ideas" (Decreto 37.215, 2013, p. 3). La oficina utiliza millones de datos generados por los organismos públicos, por empresas asociadas al proyecto (como Google, a través de Waze, Google Maps y Google Earth, y también Moovit y 99Táxi) y los ciudadanos (incluso en las redes sociales), reuniéndolos en una única base de datos, "para ayudar a las secretarías en su búsqueda de mejorar la ciudad, siempre". La aplicación Waze repasa al COR datos de sus usuarios con relatos sobre accidentes y condiciones del tránsito.

La iniciativa es defendida como parte de la creencia en un modelo de "polisdigitocracia":

Estamos seguros que, al recoger datos sobre el comportamiento de la Ciudad en sus más diversos aspectos, las 24 horas del día, 7 días a la semana, incluso cuando el ciudadano no tiene tiempo para parar, reflexionar y enviar una sugerencia, estamos ayudando en la construcción de lo que llamamos Democracia Digital Ubicua, es decir, aquella democracia que, al final está siempre presente ${ }^{6}$. (O escritório de inteligência, 2013).

Sin embargo, el decreto que creó el Pensa - Sala de Ideas en ningún momento establece una política de uso de datos de los ciudadanos. El jefe ejecutivo de operaciones del COR del 2013 al 2016, Pedro Junqueira, define smart city como:

la capacidad de la ciudad para gestionar mejor sus recursos, con más eficiencia, a partir del uso amplio de monitoreo y de cruce de informaciones, con el objetivo de mejor atender a los ciudadanos y formular políticas públicas más eficaces $^{7}$. (2014, p. 78)

6 "Temos certeza que, ao coletar dados sobre o comportamento da Cidade em seus mais diversos aspectos, 24 horas por dia, 7 dias por semana, mesmo quando o cidadão não tem tempo para parar, refletir e enviar uma sugestão, estamos ajudando na construção do que chamamos de Democracia Digital Ubíqua, ou seja, aquela democracia que, ao final está Sempre Presente".

7 “[...] a capacidade de a cidade gerir melhor seus recursos, com mais eficiência, a partir do uso amplo de monitoramento e de cruzamento de informações, com o objetivo de melhor atender aos cidadãos e formular políticas públicas mais eficazes". 
Y a continuación complementa:

A partir de mi experiencia, añado a este concepto que una ciudad inteligente es aquella capaz de integrar al ciudadano al proceso de gestión, y esa integración puede y debe ser facilitada por el uso de la tecnología. Aquí en Río, la voz del carioca suena cada vez más alta, clara y constante junto al gobierno municipal. Más que intención o tendencia, oír las calles está en el corazón de acciones y programas de la gestión municipal ${ }^{8}$. (2014, p. 78, énfasis nuestro)

En la escucha de las calles se incluye el intercambio de informaciones entre ciudadanos y gobierno, "imbuidos de objetivos alineados e interdependencia reconocida por las partes". De la parte de los residentes, por lo que informa el exjefe del COR, los datos se generan cuando usan el número de teléfono 1746 (también a través de la aplicación de celular) para requerir la prestación de servicios de varios órganos municipales, como remoción de escombros, obras públicas, fiscalización de taxis, entre varios otros, y cuando acceden a los espacios para la oferta de acceso a la informática y a la internet, donde también se realizan cursos y actividades de ocio, conocidos como Naves del Conocimiento.

Sin embargo, no queda totalmente claro cómo el Ayuntamiento, después de la asociación con Waze en el 2013, tiene acceso a los datos de los usuarios de la aplicación de la empresa comprada por Google, por más útiles que puedan ser los servicios prestados a partir de ellos, ni si Waze tiene acceso a más que las informaciones sobre las intervenciones programadas por los organismos públicos municipales, como interdicciones de calles para eventos y obras. En tiempos de robos masivos de datos de usuarios, toda transparencia en ese terreno, aún más involucrando a la administración pública, será muy bienvenida.

Probablemente no todos los cariocas saben pero, además de sus tweets sobre diferentes problemas en la ciudad, como accidentes de tránsito, caídas de árboles, protestas e incendios, el COR también identifica la ubicación de los usuarios de Twitter. Y, Junqueira (2014) también vislumbra un prontuario médico único de los habitantes, disponible en la nube, pero, aun defendiendo las ventajas del recurso como forma de salvar vidas, se anticipa en manifestar preocupación por los riesgos para la privacidad. Además de las 1500 cámaras que el COR tiene para monitoreo de la ciudad, apuesta sus fichas también en cámaras instaladas en los vehículos de la Guardia Municipal y de la Compañía de Ingeniería de Tráfico de

8 "A partir da minha experiência, acrescento a este conceito que uma cidade inteligente é aquela capaz de integrar o cidadão ao processo de gestão, e essa integração pode e deve ser facilitada pelo uso da tecnologia. Aqui no Rio, a voz do carioca soa cada vez mais alta, clara e constante junto ao governo municipal. Mais do que intenção ou tendência, ouvir as ruas está no cerne de ações e programas da gestão municipal". 
la ciudad (CET-Río), incluso en las de video analítico, capaces de "pensar por el operador" y, por lo tanto, hacer innecesario el ojo humano para identificar diferentes ocurrencias.

La defensa de las tecnologías como forma de transformación de Río en smart city pasa aún, en la visión de Junqueira, por la implantación de sistemas de e-learning en las escuelas, aunque haga la salvedad de que "el contacto existente entre alumnos y profesores en el aula es muy rica y deseable"9 (2014, p. 83). Además de las cámaras ya usadas en los cascos de servidores del Ayuntamiento que circulan por eventos y envían imágenes en tiempo real al COR, también sueña con el uso de drones para fines de monitoreo. La Internet de los Objetos, de la misma forma, está en el horizonte para alcanzar la meta de lo que llama "sensorización" de Rio. Por la parte del Ayuntamiento, los datos para los residentes se suministran a través del proyecto de open data Almacén de Datos (Armazém de Dados), reunidos en el marco de su sitio en internet. La intención, según Junqueira, es que "el interés y la curiosidad individuales produzcan soluciones para la vida cotidiana de la ciudad [...] a través de aplicaciones y de productos"10 (2014, p. 80).

El modelo de smart city puesto en práctica por Río de Janeiro debería pasar no solo por el aparato sofisticado y por el modo en que las alianzas con las empresas de tecnología se establecen, sino también, ciertamente, por la forma cómo los habitantes de la ciudad participan o se ven incluidos en el proyecto. La claridad sobre cómo se utilizan sus datos y cómo se está dando la captura de imágenes, por ejemplo, es un paso importante para esa participación. Pero las afirmaciones sobre la relevancia de la escucha de los ciudadanos en este proceso, a pesar de ser la expresión democrática del cuidado con ellos por parte de los administradores públicos, se transforman en meras generalizaciones si no van acompañadas de la explicitación de los regímenes de inclusión en que los moradores, y principalmente ellos, decidan los rumbos que quieren para sus ciudades.

El neologismo "polisdigitocracia" está detrás de la idea de Rio como smart city. Para el exalcalde Eduardo Paes, dos fenómenos recientes explican la palabra: primero, el creciente poder de las ciudades, ante la acumulación de problemas más urgentes bajo la responsabilidad de sus gobernantes, como los de educación, salud, transporte e incluso los problemas aparentemente pertenecientes a las naciones, como cambios climáticos y empleo; el segundo es la omnipresencia de la revolución digital que ha permitido a las personas conectarse y comunicarse como nunca antes fue posible. Tal escenario, según él, lleva a los ciudadanos a

9 "O contato existente entre alunos e professores na sala de aula é altamente rico e desejável".

10 "O interesse e a curiosidade individuais produzam soluções para o cotidiano da cidade [...] através de aplicações e de produtos". 
querer participar y colaborar más con sus gobiernos para estar más cerca de las instituciones y gobernantes, y no solo para participar en las elecciones. Y así define él la "polisdigitocracia":

Lo que estamos presenciando es el nacimiento de algo que llamo Polisdigitocracia. Esta es una forma de gobierno que considera la participación y la transparencia como sus piedras angulares y utiliza la tecnología como su guía. Y solo estamos al comienzo de ese viaje. La Polisdigitocracia tiene un potencial increíble para ayudar a nuestras ciudades a involucrar y entusiasmar a los ciudadanos para lograr nuestros objetivos comunes ${ }^{11}$. (Paes, 2015, p. 5)

Al invocar el concepto griego de polis, fundado en las ciudades estado donde los ciudadanos se involucran en acalorados debates sobre cuestiones públicas, para explicar el término que él mismo acuñó, Paes considera que las contiendas, antes tenidas en las ágoras, pueden ahora darse en el ambiente de internet. Es una apuesta honesta siempre que el Ayuntamiento cree verdaderamente las condiciones para que ese espacio digital sea ocupado por los ciudadanos, con participación efectiva en la construcción del modelo de desarrollo, sostenibilidad, organización, inclusión, etcétera, que quieren para Río. No siendo así, la administración solo repetirá los patrones "democráticos" de participación instituidos por las redes sociales, que podrían ser expresados en líneas generales de la siguiente forma: le ofrecemos servicios "eficaces y gratuitos" $\mathrm{y}$, a cambio, usted me concede sus datos; lo que hago con ellos -incluso para influenciar sus decisiones, sus elecciones y visiones de mundo y para ganar (mucho) dinero- no es de su incumbencia.

Todas las acciones que los administradores realizan en pro de la reducción o extinción de los problemas que la población enfrenta en el día a día de la ciudad son, en principio, altamente loables. Solo que los fines no pueden justificar los medios. La recolección y el procesamiento de los datos de los ciudadanos, la captura de imágenes de los espacios públicos a través de miles de cámaras y la cesión de informaciones públicas a empresas privadas tienen que ser fundados en la más cristalina aclaración, incluso dando garantías de que realmente los datos no identificarán individualmente a los residentes. Por más que la línea que separa lo público de lo privado sea en la actualidad cada vez más tenue, no debe imperar en las acciones de las smart cities la lógica "Big Brother" vista en los sueños futurísticos del ex jefe del COR la Ciudad Maravillosa.

11 "What we are witnessing is the birth of something I call Polisdigitocracy. This is a form of government that counts participation and transparency as its cornerstones and uses technology as its guide. And we are only at the beginning of that journey. Polisdigitocracy has incredible potential to help our cities engage and excite citizens to achieve our common goals". 


\section{Conclusión}

La palabra "polisdigitocracia" causa impacto, llama la atención. Muy probablemente están detrás de ella las buenas intenciones de quien quiere lo mejor para la ciudad, ya sea porque, como residente también será beneficiado por el progreso, o incluso porque, en un cálculo político o profesional, quedarán laureles y dividendos para una carrera de ojo en futuros cargos como gestor público o incluso para su actuación en el ambiente empresarial. Como el volumen de problemas que se agiganta en Río de Janeiro, una ciudad, como ya se ha dicho, de contrastes imposibles de pasar inadvertidos, la apuesta por la transformación en smart city es súper válida, siempre que todas las cartas no sean invertidas solo en la tecnología.

Que Río de Janeiro sea considerado por su historia y sus especificidades como un tejido urbano urdido por millones de individualidades, estas, como compuestos de lo social, imaginan y sueñan con la posibilidad de participar en la conformación del futuro de la ciudad. Las voces que dudan de la premiación de Río como primera smart city del mundo en el 2013 y las que piden la desconcentración de la ciudad en ciertas regiones, con distribución más equitativa de las condiciones de trabajo, ocio, movilidad, cultural, etcétera, entre otras demandas, ciertamente tienen mucho que decir para que la metrópoli sea no solo inteligente sino también sensible.

El conocimiento que viene de los moradores que viven las ciudades en sus placeres y en sus dolores puede ser importante también para evitar que se formen grupos de expertos que, a semejanza de los escribas y sacerdotes de las primeras ciudades sumerias, formen una casta dominante capaz de todo saber para gobernar y dictar los caminos del desarrollo de esos espacios urbanos que, a pesar de las adversidades, han atravesado los milenios.

\section{Referencias}

Bruwer, Megan. (2017). Traffic congestion statistics for Rio de Janeiro based on TomTom's historical database for 2016. Tomtom - Traffic Index. Recuperado de https://www.tomtom.com/en_gb/trafficindex/city/rio-de-janeiro

Cerqueira, D., Lima, R. S., Bueno, S., Valencia, L. I., Hanashiro, O., Machado, P. H. G., y Lima, A. S. (2017). Atlas da violência 2017. Rio de Janeiro: IPEA/Fórum Brasileiro de Segurança Pública. Recuperado de http:// www.ipea.gov.br/atlasviolencia/arquivos/downloads/8891-1250170602atlasdaviolencia2017.pdf

Childe, V. G. (1950). The urban revolution. The Town Planning Review, 21(1), 3-17. 
COR completa seis anos e lança vídeo tour de realidade virtual. (30 de diciembre de 2016). Rio de Janeiro: Prefeitura do Rio de Janeiro (site). Recuperado de http://prefeitura.rio/web/guest/exibeconteudo?id=6638288

Decreto no 37.215, de 3 de junho de 2013. (4 de junio de 2013). Cria a área de Big Data - "PENSA - SALA DE IDEIAS", no âmbito da Prefeitura da Cidade do Rio de Janeiro. Diário Oficial do Município do Rio de Janeiro, no 51, Rio de Janeiro. Recuperado de http://doweb.rio.rj.gov.br/portal/visualizacoes/ pdf/2086\#/p:3/e:2086?find=DECRETO\%20N\%C2\%BA\%2037.215

Instituto Brasileiro de Geografia e Estatística. (2010). Índice de Desenvolvimento Humano. Rio de Janeiro: IBGE. Recuperado de https://cidades.ibge.gov.br/ brasil/rj/rio-de-janeiro/pesquisa/37/30255

Instituto Brasileiro de Geografia e Estatística. (2018). Panorama. Rio de Janeiro. Recuperado de https:/cidades.ibge.gov.br/brasil/rj/rio-de-janeiro/panorama

Junqueira, P. (2014). Por dentro do Centro de Operações da Prefeitura do Rio de Janeiro. Cadernos FGV Projetos - Cidades inteligentes e mobilidade urbana. 24(9), 76-84. Recuperado de http://fgvprojetos.fgv.br/publicacao/cadernos-fgvprojetos-no-24-cidades-inteligentes-e-mobilidade-urbana

Karlgaard, R. y Malone, M. (1995). City vs. country: Tom Peters \& George Gilder debate the impact of technology on location. (Forbes ASAP columnist Tom Peters and contributing editor George Gilder). Forbes ASAP: A Technology Supplement, 155(5), 56-61.

Las 50 ciudades más violentas del mundo. (6 de abril de 2017). Ciudad de México: Seguridad, Justicia y Paz. Recuperado de https://www. seguridadjusticiaypaz.org.mx/biblioteca/prensa/send/6-prensa/242-las50-ciudades-mas-violentas-del-mundo-2017-metodologia

Leite, C. (2014). Inteligência territorial: cidades inteligentes com urbanidade. Cadernos FGV Projetos - Cidades inteligentes e mobilidade urbana, 24(9), 76-84. Recuperado de http://fgvprojetos.fgv.br/publicacao/cadernos-fgv-projetosno-24-cidades-inteligentes-e-mobilidade-urbana

Lemos, A. (2014). Cidades inteligentes: lugar, territorialização informacional $e$ inteligência. Recuperado de http://www.lab404.ufba.br/?p=2491

Lévy, P. (1993). As tecnologias da inteligência: o futuro do pensamento na era da informática. Rio de Janeiro: Editora 34.

O escritório de inteligência de dados da Prefeitura da Cidade do Rio de Janeiro: o que fazemos. (2013). Pensa - Sala de Ideias. Recuperado de http://www. pensa.rio/main/pensa/ 
Organización de las Naciones Unidas. (2014). World Urbanization Prospects: The 2014 Revision, Highlights (ST/ESA/SER.A/352). Recuperado de https:// population.un.org/wup/Publications/Files/WUP2014-Highlights.pdf

Paes, E. (2015). Foreword - Mayor Paes. Polisdigitocracy: digital technology, citizen engagement and climate action, 1,(5). Recuperado de http://www.c40.org/ researches/polisdigitocracy-digital-technology-citizen-engagement-andclimate-action

Pfaelli, M. P., Rollier, R., Vonlanthen, B., y Wade, M. (2016). Smart city: essentials for city leaders. Lausanne: International Institute for Management Development/Swisscom AG. Recuperado de https://www.imd.org/dbt/ reports/smart-city-essentials-for-city-leaders/

Rio de Janeiro é uma das 10 cidades mais congestionadas do mundo, diz pesquisa. (21 de febrero del 2017). G1. Recuperado de https://g1.globo. com/carros/noticia/mesmo-com-melhora-rio-e-uma-das-10-cidades-maiscongestionadas-do-mundo.ghtml

Townsend, A. M. (2013). Smart cities: big data, civic hackers, and the quest for a new utopia. Nueva York: W. W. Norton \& Company. 